\title{
Measurement of the pairwise kinematic Sunyaev-Zeldovich effect with Planck and BOSS data
}

DOI:

10.1103/PhysRevD.97.023514

\section{Document Version}

Final published version

Link to publication record in Manchester Research Explorer

\section{Citation for published version (APA):}

Li, Y. C., Ma, Y. Z., Remazeilles, M., \& Moodley, K. (2018). Measurement of the pairwise kinematic SunyaevZeldovich effect with Planck and BOSS data. Physical Review D, 97(2), [023514].

https://doi.org/10.1103/PhysRevD.97.023514

\section{Published in:}

Physical Review D

\section{Citing this paper}

Please note that where the full-text provided on Manchester Research Explorer is the Author Accepted Manuscript or Proof version this may differ from the final Published version. If citing, it is advised that you check and use the publisher's definitive version.

\section{General rights}

Copyright and moral rights for the publications made accessible in the Research Explorer are retained by the authors and/or other copyright owners and it is a condition of accessing publications that users recognise and abide by the legal requirements associated with these rights.

\section{Takedown policy}

If you believe that this document breaches copyright please refer to the University of Manchester's Takedown Procedures [http://man.ac.uk/04Y6Bo] or contact uml.scholarlycommunications@manchester.ac.uk providing relevant details, so we can investigate your claim.

\section{OPEN ACCESS}




\title{
Measurement of the pairwise kinematic Sunyaev-Zeldovich effect with Planck and BOSS data
}

\author{
Yi-Chao Li, ${ }^{1,2}$ Yin-Zhe Ma, ${ }^{1,2,3, *}$ Mathieu Remazeilles, ${ }^{3}$ and Kavilan Moodley ${ }^{4,2}$ \\ ${ }^{1}$ School of Chemistry and Physics, University of KwaZulu-Natal, Westville Campus, \\ Private Bag X54001, Durban 4000, South Africa \\ ${ }^{2}$ NAOC-UKZN Computational Astrophysics Centre (NUCAC), \\ University of KwaZulu-Natal, Durban 4000, South Africa \\ ${ }^{3}$ Jodrell Bank Centre for Astrophysics, Alan Turing Building, School of Physics and Astronomy, \\ The University of Manchester, Oxford Road, Manchester M13 9PL, United Kingdom \\ ${ }^{4}$ Astrophysics and Cosmology Research Unit, School of Mathematics. Statistics and Computer Science, \\ University of KwaZulu-Natal, Durban 4041, South Africa
}

(Received 31 October 2017; published 18 January 2018)

\begin{abstract}
We present a new measurement of the kinetic Sunyaev-Zeldovich effect (kSZ) using Planck cosmic microwave background (CMB) and Baryon Oscillation Spectroscopic Survey (BOSS) data. Using the "LowZ North/South" galaxy catalogue from BOSS DR12, and the group catalogue from BOSS DR13, we evaluate the mean pairwise kSZ temperature associated with BOSS galaxies. We construct a "Central Galaxies Catalogue" (CGC) which consists of isolated galaxies from the original BOSS data set, and apply the aperture photometry (AP) filter to suppress the primary CMB contribution. By constructing a halo model to fit the pairwise kSZ function, we constrain the mean optical depth to be $\bar{\tau}=$ $(0.53 \pm 0.32) \times 10^{-4}(1.65 \sigma)$ for LowZ North CGC, $\bar{\tau}=(0.30 \pm 0.57) \times 10^{-4}(0.53 \sigma)$ for LowZ South CGC, and $\bar{\tau}=(0.43 \pm 0.28) \times 10^{-4}(1.53 \sigma)$ for DR13 Group. In addition, we vary the radius of the AP filter and find that the AP size of 7 arcmin gives the maximum detection for $\bar{\tau}$. We also investigate the dependence of the signal with halo mass and find $\bar{\tau}=(0.32 \pm 0.36) \times 10^{-4}(0.8 \sigma)$ and $\bar{\tau}=$ $(0.67 \pm 0.46) \times 10^{-4}(1.4 \sigma)$ for DR13 Group with halo mass restricted to, respectively, less and greater than its median halo mass, $10^{12} h^{-1} \mathrm{M}_{\odot}$. For the LowZ North CGC sample restricted to $M_{\mathrm{h}} \gtrsim 10^{14} h^{-1} \mathrm{M}_{\odot}$ there is no detection of the $\mathrm{kSZ}$ signal because these high mass halos are associated with the high-redshift galaxies of the LowZ North catalogue, which have limited contribution to the pairwise kSZ signals.
\end{abstract}

DOI: 10.1103/PhysRevD.97.023514

\section{INTRODUCTION}

The kinematic Sunyaev-Zeldovich effect (hereafter, kSZ effect), first proposed in [1,2], describes the anisotropy of the CMB due to its scattering off moving electrons in the Universe,

$$
\frac{\Delta T}{T}=-\frac{\sigma_{\mathrm{T}}}{c} \int \mathrm{d} \ln _{\mathrm{e}}(\mathbf{v} \cdot \hat{\mathbf{n}}),
$$

where $\sigma_{\mathrm{T}}$ is the Thomson cross section, $n_{\mathrm{e}}$ is the electron density and $\mathbf{v}$ is the peculiar velocity of electrons relative to the CMB. In the limit of nonrelativistic elastic scattering (Thomson scattering), the kSZ effect is equally efficient for all frequencies and causes a frequency-independent distortion of CMB spectrum.

In recent years, there have been a series of works to detect and measure the kSZ signal. Hand et al. [3] first reported the detection of the kSZ signal by applying the

*Ma@ukzn.ac.za pairwise $\mathrm{kSZ}$ estimator [4] to CMB data from the Atacama Cosmology Telescope (ACT [5]) using a galaxy catalog from the Sloan Digital Sky Survey III DR9 [6]. Recently, this measurement of the pairwise $\mathrm{kSZ}$ effect was achieved with higher precision using ACT CMB data combined with the BOSS DR11 catalogue [7]. With the Planck map, Planck Collaboration et al. [8] reported a kSZ detection using the pairwise kSZ estimator on the Central Galaxy Catalogue (CGC) extracted from SDSS DR7 [9]. Reference [8] also constructed the correlation function between the peculiar velocity field and kSZ temperature anisotropies and achieved the first detection at $3.0 \sigma$ confidence level (C.L.). Apart from the use of spectroscopic surveys, Soergel et al. [10] detected the pairwise kSZ effect with photometric survey data from the Dark Energy Survey (DES [11]) and CMB data from the South Pole Telescope (SPT [12]).

In addition to the pairwise temperature difference estimator of the kSZ effect, there have been several other statistical methods developed to measure the kSZ effect. 
Sugiyama et al. [13] proposed the density-weighted pairwise $\mathrm{kSZ}$ estimator in Fourier space, and achieved a $2.54 \sigma$ detection with the Planck CMB map and BOSS DR12 catalogue [14]. Hill et al. [15] and Ferraro et al. [16] crosscorrelated the squared temperature map from Planck and Wilkinson Microwave Anisotropy Probe (WMAP [17]) data, appropriately filtered to isolate the small-scale $\mathrm{kSZ}$ signal, with the projected galaxy positions from the Widefield Infrared Survey Explorer (WISE [18]) and achieved a $\sim 4.0 \sigma$ C.L. detection of the kSZ effect. More recently, Planck Collaboration et al. [19] detected the velocity dispersion effect of the $\mathrm{kSZ}$ signal from the Planck 2D-ILC CMB map at $3.2 \sigma$ C.L.

As the measurement of the $\mathrm{kSZ}$ effect becomes more accurate, there is the potential to use it to trace the peculiar velocities of galaxies and clusters. The pairwise kSZ signal is closely related to the pairwise velocity of the galaxies or clusters, which encodes information about large-scale structure. Previous studies showed that the kSZ measurement can be used to constrain the dark energy equation of state [20-25] and modified gravity models [26]. Recently, Hernández-Monteagudo et al. [27] showed that the baryon fraction is consistent with unity for the kSZ-peculiar velocity field cross-correlation signal. In addition, Mueller et al. [28] pointed out that the kSZ effect can be used to constrain neutrino mass with precision measurements and large enough samples.

In this work, we focus on measuring the pairwise $\mathrm{kSZ}$ signals using the foreground-cleaned Planck 2D-ILC CMB map, which, by construction, is free from thermal SZ (tSZ) residual contamination [29], and the galaxy and group catalogues from BOSS DR12 and DR13. In Sec. II, we present the details of the CMB map and the galaxy and group catalogues that we use. The estimator and theoretical model are introduced in Secs. III and IV, respectively. We summarize and discuss our results in Sec. V. In our analysis, we adopt a spatially flat $\Lambda \mathrm{CDM}$ cosmology model with the cosmological parameters fixed to the best-fit values of Planck Collaboration et al. [30]: $\Omega_{\mathrm{m}}=0.309, \Omega_{\Lambda}=0.691$, $n_{\mathrm{s}}=0.9608, \sigma_{8}=0.815$ and $h=0.68$.

\section{DATA}

\section{A. The Planck 2D-ILC CMB map}

In this work, we use the $2 \mathrm{D}$ - ILC CMB map, which we have obtained by applying the 'Constrained ILC' component separation method [29] to the public Planck 2015 data. ${ }^{1}$ The 'Constrained ILC' method is specifically designed to nullify the residuals of thermal SunyaevZeldovich (tSZ) emission in the CMB map, while minimizing the contamination from other foregrounds and noise. Along similar lines to those of the standard NILC method [31,32], the 'Constrained ILC' approach performs

\footnotetext{
${ }^{1}$ http://pla.esac.esa.int/pla.
}

a minimum-variance weighted linear combination of the nine Planck frequency maps, with the weights giving unit response to the CMB spectral energy distribution. However, the 'Constrained ILC' method offers an additional constraint for the vector of weights to be orthogonal to the spectral energy distribution of the tSZ effect, thus providing a null response to the tSZ signal during the filtering. As a result, the so-called 2D-ILC CMB map, where 2D stands for the two-dimensional constraint on $\mathrm{CMB}$ and tSZ, is free from tSZ residuals in the direction of the galaxy clusters, unlike other CMB maps released by Planck. Therefore, the 2D-ILC CMB map allows us to probe the $\mathrm{kSZ}$ effect in the direction of galaxy clusters without suffering from tSZ bias and variance. The fullwidth-half-maximum (FWHM) of the 2D-ILC map is $\theta_{\mathrm{FWHM}}=5$ arcmin. For more details on the constrained ILC method and on the 2D-ILC CMB map, we refer to [29] and Sec. II. A of Planck Collaboration et al. [19].

\section{B. Catalogue of galaxies}

\section{Central galaxy catalogue}

The galaxy samples in the Central Galaxy Catalogue (CGC) are selected to trace the centres of dark matter halos. We follow the method used in Ref. [8] to find isolated galaxies with no other galaxies within $1.0 \mathrm{Mpc}$ in the transverse direction and with a redshift difference smaller than $1000 \mathrm{~km} \mathrm{~s}^{-1}$. Such an isolation criterion is applied to the large-scale structure galaxy catalogue of the twelfth data release of the Baryon Oscillation Spectroscopic Survey (BOSS DR12). The BOSS sample was designed to measure the $\mathrm{BAO}$ signature in the two-point galaxy clustering statistical analysis, and separated into 'LOWZ' and 'CMASS' (for 'Constant (stellar) Mass') catalogues [33]. Both LOWZ and CMASS include two separated survey areas, located in the Northern and Southern Galactic caps, respectively. We use LOWZ North/South as the labels for the two different LOWZ subcatalogues. After applying the isolation criterion, around $20 \%$ of the galaxies are flagged out for LOWZ North/South catalogue. The redshift histograms for the LOWZ North/South CGC are shown in Fig. 1, and the total number of galaxies in each catalogue are listed in the legend. Figure 1 also shows the redshift distribution of the CGC selected from SDSS DR7 with label DR7 CGC, which is used in the kSZ analysis of Ref. [8]. After applying the CGC criterion, the sample amount of the LOWZ North CGC is comparable to that of DR7 CGC, but the LOWZ North CGC samples, as well as the LOWZ South CGC samples, have broad redshift distributions with a median value of $z_{\text {median }}=0.315$. The LowZ sample is designed to extend the SDSS-I/II Luminous Red Galaxy (LRG) sample to higher redshift and fainter luminosities. But it also includes a bright magnitude cut, which excludes a large number of the low-redshift galaxies. We will discuss how the magnitude cut affects the kSZ signals in Sec. V. 


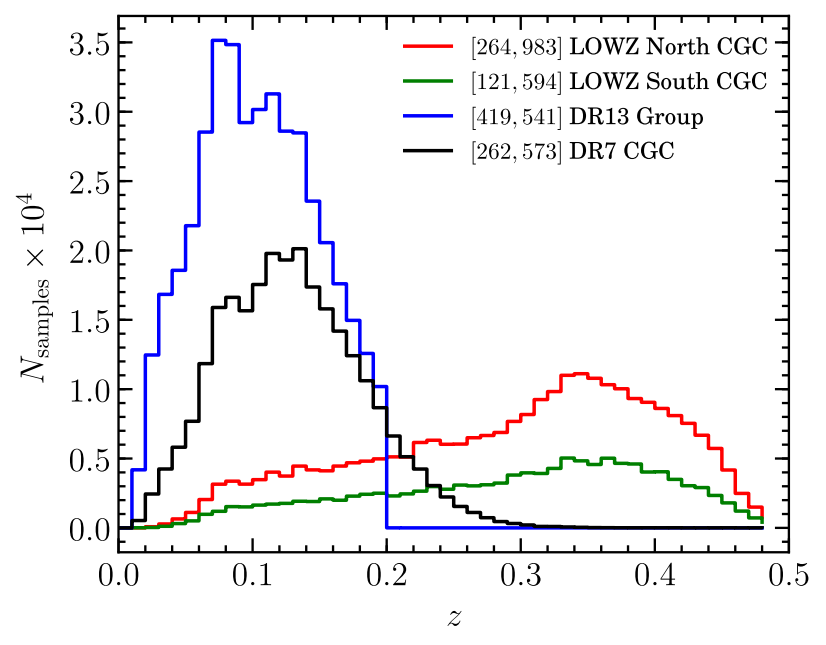

FIG. 1. The redshift histogram for each catalogue. The total number of galaxies in each catalogue is shown in the legend. The DR7 CGC catalogue that was used in Planck Collaboration et al. [8] is also shown here as the black curve.

\section{Group catalogue}

We also analyze the recently updated SDSS group catalogue [34], which is constructed with the galaxy samples from the SDSS DR13 Northern Galactic Cap galaxy catalogue. The redshift histogram of the SDSS group catalogue is shown as a blue line in Fig. 1. The galaxies with $z>0.2$ are cut out in this catalogue [8]. The group catalogue has a similar redshift distribution as the DR7 CGC, which is the central galaxy catalogue used in the previous analysis of [8].

The group finder used for the DR13 Group catalogue is based on that of Ref. [35], with improved halo mass assignment. The improved group finder gives more accurate halo-mass estimates, and extends the group samples to even lower mass.

The number of samples, sky coverage and halo mass and redshift range of LowZ North and South catalogue, and the DR13 group catalogue are shown in Table I.

\section{THE ESTIMATOR}

The CMB brightness temperature fluctuation induced by the kSZ effect of a galaxy cluster is given by

$$
\delta T_{\mathrm{kSZ}, i}=-\frac{T_{0} \bar{\tau}}{c} \mathbf{v}_{i} \cdot \hat{\mathbf{n}}_{i},
$$

TABLE I. Summary of the catalogue information.

\begin{tabular}{lccc}
\hline \hline Catalogue & LowZ North & LowZ South & Group \\
\hline$N_{\text {sample }}$ & CGC & CGC & DR13 \\
$S_{\text {overlap }}\left[\mathrm{deg}^{2}\right]$ & 264983 & 121594 & 419541 \\
$z$ & $\sim 5600$ & $\sim 2500$ & $\sim 6900$ \\
$\log _{10}\left(M_{\mathrm{h}} / h^{-1} \mathrm{M}_{\odot}\right)$ & {$[0.01,0.50]$} & {$[0.01,0.50]$} & {$[0.01,0.20]$} \\
\hline \hline
\end{tabular}

where $\bar{\tau}$ is the mean optical depth of the sample, $T_{0}=$ $2.725 \mathrm{~K}$ is the mean CMB temperature, $c$ is the speed of light, $\hat{\mathbf{n}}_{i}$ is the line-of-sight direction, and $\mathbf{v}_{i}$ is the peculiar velocity of the galaxy cluster relative to the CMB. Here we make the assumption that the gas in the cluster traces the mass, and that all clusters have the same gas-mass fraction. We also assume that the peculiar velocity of free electrons is the same as the velocity of dark matter, so that it traces the underlying dark matter distribution.

The kSZ effect of a single galaxy cluster is known to be an order of magnitude lower than the tSZ effect and emission from dusty star-forming galaxies, thus the direct measurement of the single-cluster $\mathrm{kSZ}$ effect is challenging. However, the kSZ effect due to the relative movement between the cluster pairs has been shown to be detectable $[3,7,8]$. The pairwise momentum estimator is defined as $[3,4]$

$$
\hat{p}_{\mathrm{kSZ}}(r)=-\frac{\sum_{i<j}\left(\delta T_{\mathrm{kSZ}, i}-\delta T_{\mathrm{kSZ}, j}\right) c_{i j}}{\sum_{i<j} c_{i j}^{2}},
$$

in which $i$ and $j$ indicate a pair of clusters, and $c_{i j}$ is the weight factor which only depends on the geometry of the pair

$$
c_{i j}=\frac{\left(r_{i}-r_{j}\right)(1+\cos \theta)}{2 \sqrt{r_{i}^{2}+r_{j}^{2}-2 r_{i} r_{j} \cos \theta}},
$$

with $r_{i}$ and $r_{j}$ being the comoving distance of the two galaxies or clusters, and $\theta$ being the angular separation between the two galaxies or clusters, i.e. $\cos \theta=\hat{\mathbf{r}}_{i} \cdot \hat{\mathbf{r}}_{j}$. If the sample size is large enough any signal that is independent of the comoving separation will be averaged to zero, due to the differential estimation. However, the infrared emission of the galaxies and the tSZ signal may have small redshift-dependent variation, due to the cosmic evolution of the cluster mass and temperature. The estimator can not avoid the contamination of the redshiftdependent signals. Therefore, Refs. [3,7,8] define the following Gaussian weighted average temperature of the sources as a function of redshift, so that by subtracting it the redshift-dependent variation will be removed

$$
\mathcal{T}(z)=\frac{\sum_{i} T_{i} \exp \left[-\left(z-z_{i}\right)^{2} / \sigma_{z}^{2}\right]}{\sum_{i} \exp \left[-\left(z-z_{i}\right)^{2} / \sigma_{z}^{2}\right]}
$$

where $T_{i}$ is the temperature associated with the $i$ th sample and $z_{i}$ is the redshift center of the $i$ th sample. The choice of $\sigma_{z}$ has no significant effect on the measurements [3,7,8]. In this work, we use $\sigma_{z}=0.001$. Then,

$$
\delta T_{\mathrm{kSZ}, i}=T_{i}-\mathcal{T}\left(z_{i}\right)
$$

In order to extract $T_{i}$, we apply the aperture photometry (AP) filter to the CMB map. The main advantage of the AP 
filter is that it is independent of any assumptions of the halo profile. The size of the AP filter can affect the detection of the $\mathrm{kSZ}$ pairwise momentum. The details of the AP filter are discussed further in Sec. V.

\section{MODEL FITTING}

\section{A. Mean pairwise velocity}

The mean pairwise velocity between pairs of dark matter halos separated with comoving distance $r$ can be expressed with the two-point correlation function of dark matter using the pair conservation equation $[23,36]$,

$$
v(r, a(z))=-\frac{2}{3} H(a) a f(a) \frac{r^{\overline{g h a l o}^{\circ}}(r, a)}{1+\xi^{\text {halo }}(r, a)},
$$

where $a$ is the scale factor of the universe, $\xi^{\text {halo }}(r, a)$ is the two-point correlation function,

$$
\xi^{\text {halo }}(r, a)=\frac{1}{2 \pi^{2}} \int_{0}^{\infty} \mathrm{d} k k^{2} j_{0}(k r) P(k, a) b_{\text {halo }}^{(2)}(k, a),
$$

where $j_{0}$ is the zero order spherical Bessel function, and $P(k ; a)$ is the linear matter power spectrum at scale factor $a$. Here $\xi^{\text {halo }}(r, a)$ is the two-point correlation function averaged over a sphere of radius $r$,

$$
\begin{aligned}
\bar{\xi}^{\text {halo }}(r, a)= & \frac{3}{2 \pi^{2} r^{3}} \int_{0}^{r} \mathrm{~d} r^{\prime} r^{2} \\
& \times \int_{0}^{\infty} \mathrm{d} k k^{2} j_{0}\left(k r^{\prime}\right) P(k, a) b_{\text {halo }}^{(1)}(k, a) .
\end{aligned}
$$

The halo bias factor, $b_{\text {halo }}^{(1)}(k, a)$ and $b_{\text {halo }}^{(2)}(k, a)$ are given by $b_{\text {halo }}^{(q)}(k, z)=\frac{\int \mathrm{d} M M(\mathrm{~d} n / \mathrm{d} M) b(M, z)^{q} W^{2}(k R(M))}{\int \mathrm{d} M M(\mathrm{~d} n / \mathrm{d} M) W^{2}(k R(M))}$.

We take the expression of $b(M, z)$ in Ref. [37],

$$
b(M, z)=1+\frac{\delta_{\mathrm{c}}^{2}-\sigma^{2}(M, z)}{\sigma^{2}(M, z) \delta_{\mathrm{c}}},
$$

where $\delta_{\mathrm{c}}=1.686$, and $\sigma^{2}(M, z)$ is the rms fluctuation on a mass scale $M$ at redshift $z$

$$
\sigma^{2}(M, z)=\frac{1}{2 \pi^{2}} \int_{0}^{\infty} \mathrm{d} k k^{2} P(k, z) W^{2}(k R(M)) .
$$

The halo mass function (HMF) takes the form

$$
\frac{\mathrm{d} n}{\mathrm{~d} M}(M, z)=f(\sigma) \frac{\bar{\rho}_{\mathrm{m}}(z)}{M} \frac{\mathrm{d} \ln \sigma^{-1}}{\mathrm{~d} M},
$$

in which $\bar{\rho}_{\mathrm{m}}(z)=\Omega_{\mathrm{m}} \rho_{\text {crit }}(1+z)^{3}$ is the mean matter density of the Universe at redshift $z$ and $\rho_{\text {crit }}=2.775 h^{2} \times$ $10^{11} \mathrm{M}_{\odot} \mathrm{Mpc}^{-3}$ is the critical density of the Universe today. The collapse fraction $f(\sigma)$ has different expressions for different models. Here we use the parameterized $f(\sigma)$ and the fitting values of the parameters given by Tinker et al. [38],

$$
\begin{aligned}
f(\sigma, z) & =\alpha\left[1+\left(\beta(z) \frac{\delta_{\mathrm{c}}}{\sigma}\right)^{-2 \phi(z)}\right]\left(\frac{\delta_{\mathrm{c}}}{\sigma}\right)^{2 \eta(z)} \exp \left[-\frac{\gamma(z) \delta_{\mathrm{c}}^{2}}{2 \sigma^{2}}\right] \\
\beta(z) & =\beta(1+z)^{0.20} \\
\phi(z) & =\phi(1+z)^{-0.08} \\
\eta(z) & =\eta(1+z)^{0.27} \\
\gamma(z) & =\gamma(1+z)^{-0.01}
\end{aligned}
$$

where $\alpha=0.368, \beta=0.589, \gamma=0.864, \phi=-0.729$ and $\eta=-0.243$ [38].

The pairwise velocity modeled by Eq. (7) is based on linear perturbation theory and several assumptions, such as pair conservation of galaxies (halos), isotropic peculiar velocities, and isotropic clustering of galaxies (halos), which are not valid in redshift space. Ref. [14] pointed out that the Kaiser effect from the redshift space distortions (RSDs) increases the amplitude of the pairwise kSZ signal by $\sim 20 \%$. Moreover, at nonlinear scales $\left(r<20 h^{-1} \mathrm{Mpc}\right)$, the sign of the pairwise velocity function changes from negative to positive at a scale around $10 h^{-1} \mathrm{Mpc}[7,13,39]$. Therefore, in our analysis, we restrict our model fitting to scales $>20 h^{-1} \mathrm{Mpc}$.

\section{B. Average optical depth}

The mean pairwise momentum is related to the mean pairwise velocity by a scaling factor,

$$
p_{\mathrm{kSZ}}(r, a)=\bar{\tau} \frac{T_{\mathrm{CMB}}}{c} v(r, a)
$$

where $\bar{\tau}$, as a free parameter, can be interpreted as the mean optical depth within the solid angle of the AP filter size, averaged over the cluster samples. We perform a $\chi^{2}$ minimization to find the best-fitting values for $\bar{\tau}$,

$$
\chi^{2}=\sum_{i, j}\left(p_{\mathrm{kSZ}}^{\mathrm{est}}\left(r_{i}\right)-\bar{\tau} p_{\mathrm{kSZ}}^{\mathrm{th}}\left(r_{i}\right)\right) C_{i j}^{-1}\left(p_{\mathrm{kSZ}}^{\mathrm{est}}\left(r_{j}\right)-\bar{\tau} p_{\mathrm{kSZ}}^{\mathrm{th}}\left(r_{j}\right)\right),
$$

where $p_{\mathrm{kSZ}}^{\text {est }}\left(r_{i}\right)$ is the estimated kSZ momentum in the $i$ th separation bin, $p_{\mathrm{kSZ}}^{\text {th }}\left(r_{i}\right)=\left(T_{\mathrm{CMB}} / c\right) v\left(r_{i}\right)$ is the theoretical predictions of the kSZ momentum (Eq. (7)), and $C_{i j}$ denotes the $i j$ component of the covariance matrix. The 
estimation of covariance matrix is discussed in Sec. VA. The best-fit value of $\bar{\tau}$ and its associated error are:

$$
\begin{aligned}
\bar{\tau} & =\frac{\sum_{i, j} p_{\mathrm{kSZ}}^{\mathrm{est}}\left(r_{i}\right) C_{i j}^{-1} p_{\mathrm{kSZ}}^{\mathrm{th}}\left(r_{j}\right)}{\sum_{i, j} p_{\mathrm{kSZ}}^{\mathrm{th}}\left(r_{i}\right) C_{i j}^{-1} p_{\mathrm{kSZ}}^{\mathrm{th}}\left(r_{j}\right)} \\
\sigma_{\bar{\tau}}^{2} & =\frac{1}{\sum_{i, j} p_{\mathrm{kSZ}}^{\mathrm{th}}\left(r_{i}\right) C_{i j}^{-1} p_{\mathrm{kSZ}}^{\mathrm{th}}\left(r_{j}\right)} .
\end{aligned}
$$

\section{RESULTS \& DISCUSSION}

\section{A. Correlation matrix and covariance matrix}

We first implement CMB mock samples including the thermal noise to estimate the covariance matrix. The mock samples have the advantage that they are independent of each other and properly include the cosmic variance contributed by the CMB. We generate 200 mock maps with pure $\mathrm{CMB}$ fluctuations and thermal noise and run the estimator introduced above on each of the mock maps, using the source coordinates from the real catalogue. Because there is no kSZ signal correlated with the sources, this method provides a null test for our estimator, and the estimated covariance matrix has no contribution from the measurement variance of the signal.

Another method for estimating the covariance matrix is to use jackknife samples. The jackknife sampling method [40] is widely used in the covariance matrix estimation for large-scale structure surveys. Following the jackknife method, the total survey area is divided into $N$ subareas with similar effective area. In order to reduce the correlation between jackknife samples, the subareas need to be large enough to include the modes at the scales we study. For the LowZ North (South) survey area, we have 216 (90) subareas in total with each about $25 \mathrm{deg}^{2}$. The partition is shown in Fig. 2. The survey area for 'DR13 Group' is similar to LowZ North and divided into 242 subareas with $\sim 25 \mathrm{deg}^{2}$ each. By dropping one subarea at a time, we estimate the mean

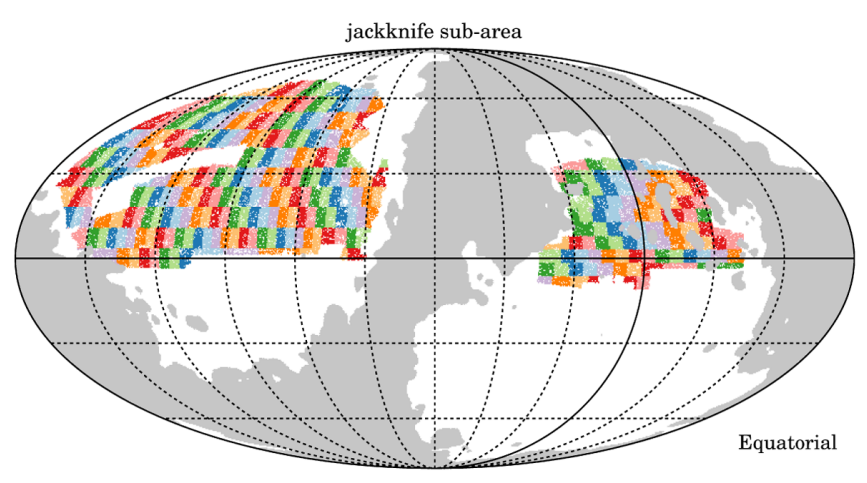

FIG. 2. The jackknife subarea division for the 'LowZ North' and 'LowZ South' survey area shown in equatorial coordinates. The grey area shows the masked range of the Planck 2D-ILC CMB map. pairwise momentum using the remaining $N-1$ subareas and obtain $N$ realizations. The covariance matrix is then estimated from the jackknife samples as

$$
C_{i j}^{\mathrm{JK}}=\frac{N-1}{N} \sum_{k=1}^{N}\left(p_{i}^{k}-\bar{p}_{i}\right)\left(p_{j}^{k}-\bar{p}_{j}\right),
$$

in which $i$ and $j$ are the indices of the comoving separation bins and $p^{k}$ is the pairwise momentum estimated using the $k$ th jackknife sample. We also apply the Hartlap factor [41] to correct the biased inverse covariance matrix of jackknife samples

$$
C^{-1}=\frac{N-N_{\mathrm{bin}}-2}{N-1} C_{\mathrm{JK}}^{-1}
$$

The correlation coefficient matrix is estimated via the covariance matrix

$$
R_{i j}=\frac{C_{i j}}{\sqrt{C_{i i} C_{j j}}} .
$$

The covariance matrices for the 'LowZ North CGC' samples are shown in the upper panels of Fig. 3, with the left panel showing the jackknife covariance and the right panel showing the CMB mock covariance. The LowZ South CGC and DR13 Group samples exhibit similar patterns in covariance matrix, but 'LowZ South CGC' has slightly higher values due to the smaller survey volume, and 'DR13 Group' has slightly lower values due to larger samples. The lower panel of Fig. 3 shows the correlation coefficient estimated with jackknife samples (left panel) and CMB mock samples (right panel). The higher correlations at larger comoving separations are recovered with both jackknife and mock samples. The red and black step lines in Fig. 4 show the diagonal error of the pairwise momentum in each bin, which is the square-root of the diagonal terms of the jackknife and CMB mock covariance matrices, estimated with 'LowZ North CGC' samples. The errors are slightly underestimated with the $\mathrm{CMB}$ mock samples, particularly at small separations, due to the lack of the simulated kSZ signals, which would add measurement variance if included. The green and blue step lines show the errors estimated with jackknife samples of 'LowZ North CGC' and 'DR13 Group', respectively. We will use the covariance matrix and diagonal errors estimated from jackknife samples for the rest of the analysis in this paper.

\section{B. Null tests}

In order to confirm that there is no systematic error in the maps or the analysis, we perform several null tests. As mentioned above, the CMB mock maps that we used for the covariance matrix estimation, which contain no kSZ signal, provide a set of maps with which we can perform a null test. By applying the estimator to the $200 \mathrm{CMB}$ mock maps, the fit 

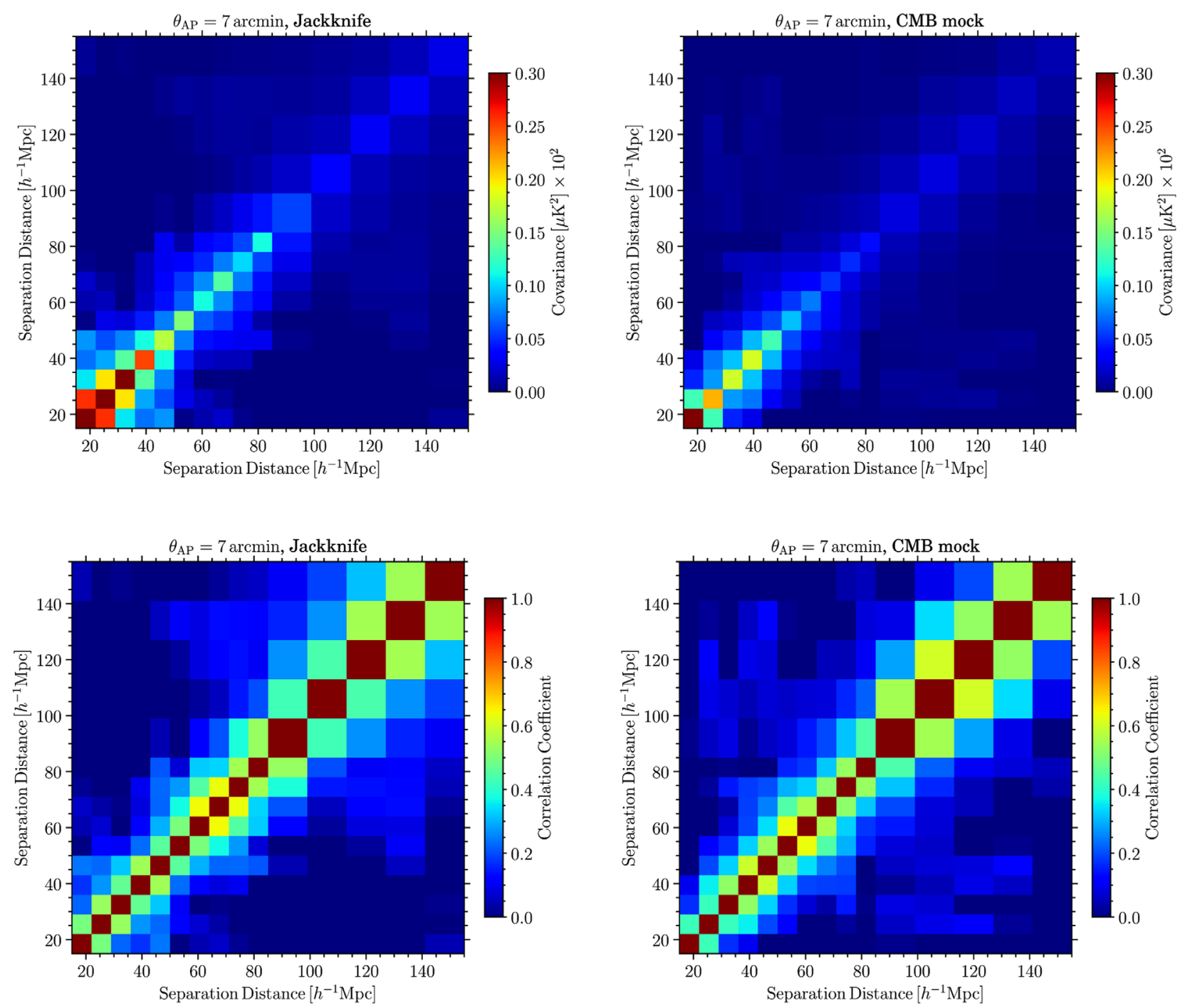

FIG. 3. The covariance matrix (the upper panels) and correlation matrix (the lower panels) for LowZ North CGC. The left panels show the covariance matrix estimated with the jackknife method, and the right panels show the covariance matrix estimated with CMB mock samples.

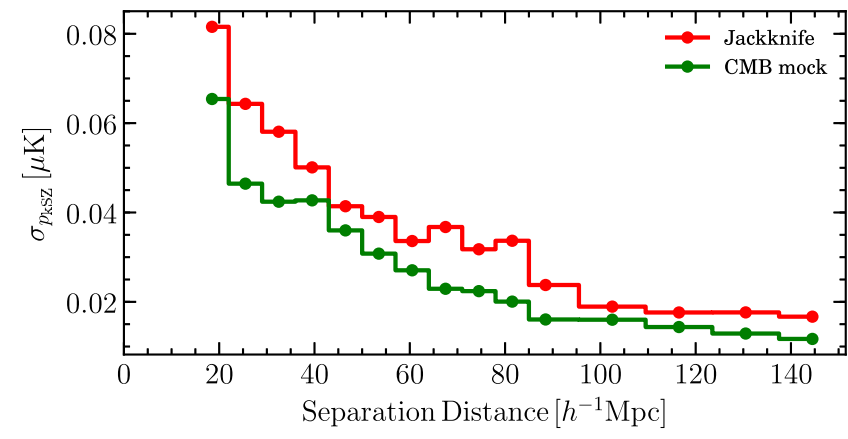

FIG. 4. The errors estimated with the jackknife (red) and CMB mock (black) samples for 'LowZ North CGC'. The green and blue step lines show the errors estimated with jackknife samples of 'LowZ North CGC' and 'DR13 Group', respectively.

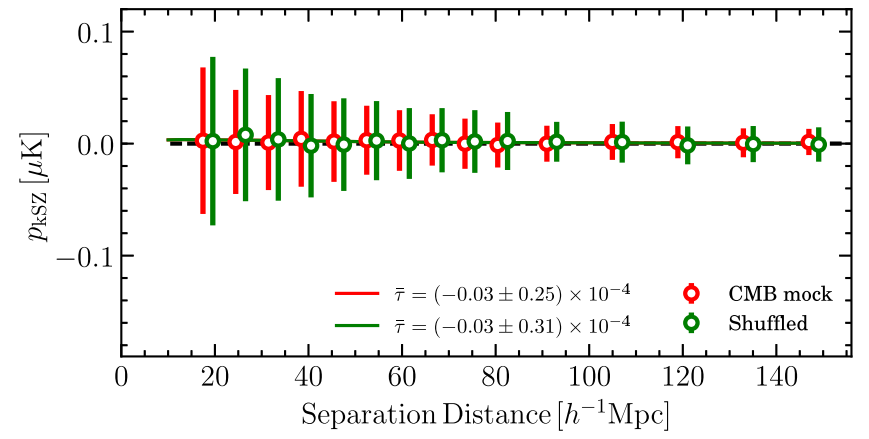

FIG. 5. Results of the null test with shuffled (green) and mock (red) CMB maps. 
to the averaged signal gives $\bar{\tau}_{\text {null }}=(0.03 \pm 0.25) \times 10^{-4}$, which is consistent with zero within $1 \sigma$ C.L. The results are shown as the red points with error bars in Fig. 5.

Another null test involves randomly shuffling the 2DILC CMB map, and applying the estimator to the shuffled maps. By averaging these realizations, the signal is similar to the CMB mock samples, $\bar{\tau}_{\text {null }}=(0.03 \pm 0.31) \times 10^{-4}$, which is also consistent with no signal. The results are shown as the green points with error bars in Fig. 5.

\section{The effect of AP filter size}

The optical depth is related to the detailed electron density profile of the dark matter halo. Due to the AP

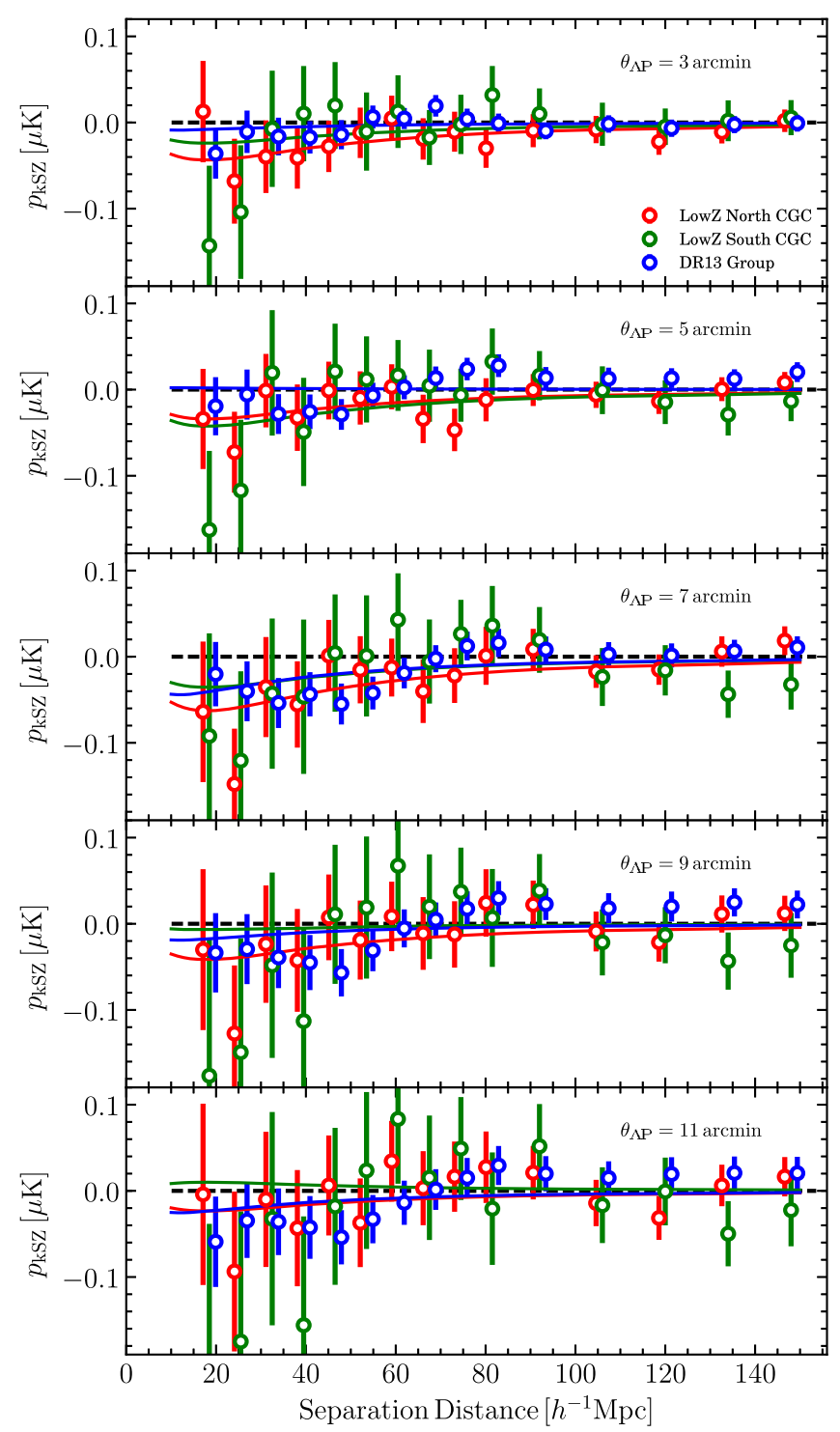

FIG. 6. The measured $p_{\mathrm{kSZ}}$ with different AP sizes. From top to the bottom, the AP radius is 3, 5, 7, 9 and 11 arcmin. The error bars shown are the square-root of the diagonal terms of the jackknife covariance matrix.

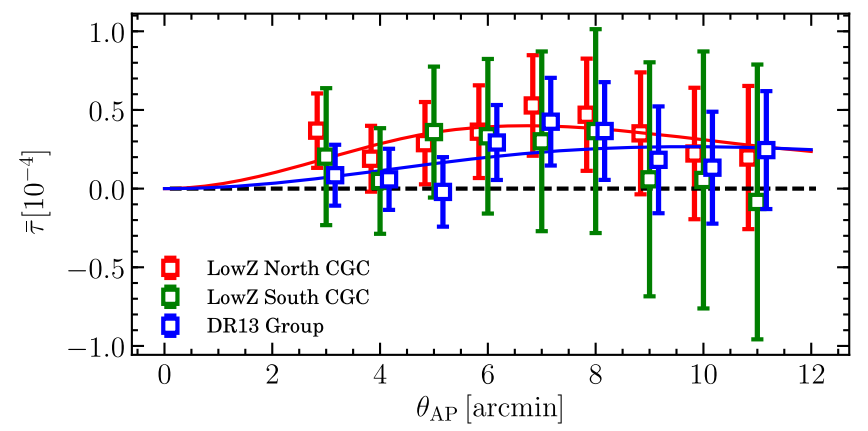

FIG. 7. The best-fit $\bar{\tau}\left(\theta_{\mathrm{AP}}\right)$ and its $1 \sigma$ errors with different AP size. The solid lines show the best-fit theoretical prediction of the mean optical depth as a function of $\theta_{\mathrm{AP}}$.

filter, the measured optical depth, $\bar{\tau}$, is the average value within the filter size. As shown in [8], the measurement can achieve the maximum detection if the filter size matches the average angular size of the gas profile of dark matter halos.

We measured the $p_{\mathrm{kSZ}}$ functions with different AP filter sizes. The $\mathrm{AP}$ radius, $\theta_{\mathrm{AP}}$, is varied from 3 arcmin, which is approaching the resolution limit of the Planck CMB map, to 11 arcmin. The results with AP radii of 3, 5, 7, 9 and 11 arcmin are shown in Fig. 6. The red, green and blue data points show the results of the LowZ North CGC, LowZ South CGC and DR13 Group catalogues, respectively. The errors shown in the figure for the different bins are the square-root of the diagonal terms of the jackknife covariance matrix. Due to the smaller survey area and the sample size, the measurement with the 'LowZ South CGC' catalogue has larger variance. The theoretical predictions, shown as the solid line in Fig. 6 are fitted to the measurements by minimizing the $\chi^{2}$ equation in Eq. (16). The fitted values of $\bar{\tau}$ with different AP radii are listed in Table II and also shown as a function of $\theta_{\mathrm{AP}}$ in Fig. 7. A significant $\theta_{\mathrm{AP}}$ dependence can be found in these the measurements. The measured value of $\bar{\tau}$ peaks at an AP radius of 7 arcmin, which is consistent with the detection in Fourier-space analysis [14], and decreases significantly when the AP radius exceeds 8 arcmin.

The mean optical depth as a function of AP radius can be modelled as [14],

$\bar{\tau}_{\mathrm{m}}\left(\theta_{\mathrm{AP}}\right)=\frac{\sigma_{\mathrm{T}} f_{\mathrm{gas}} M_{\mathrm{h}}}{\mu_{\mathrm{e}} m_{\mathrm{p}} D_{\mathrm{A}}^{2}\left(z_{\mathrm{eff}}\right)} \int \frac{\mathrm{d}^{2} \vec{\ell}}{(2 \pi)^{2}} U\left(\ell \theta_{\mathrm{AP}}\right) N(\vec{\ell}) B(\vec{\ell})$,

in which, $\sigma_{\mathrm{T}}=0.665 \times 10^{-24} \mathrm{~cm}^{2}$ is the Thomson cross section, $\mu_{\mathrm{e}}=1.14$ is the mean electron weight, $m_{\mathrm{p}}$ is the proton mass, $f_{\text {gas }}=\Omega_{\mathrm{b}} / \Omega_{\mathrm{m}} \simeq 0.155$ is the cosmic mean fraction of gas, $M_{\mathrm{h}}$ is the halo mass and $D_{\mathrm{A}}\left(z_{\text {eff }}\right)$ is the angular distance at the effective redshift.

$U\left(\ell \theta_{\mathrm{AP}}\right)$ expresses the AP filter function in Fourier space. The real space AP filter function can be modelled as a step function, 
TABLE II. The best-fit $\bar{\tau}$ with different AP radii.

\begin{tabular}{|c|c|c|c|c|c|c|c|c|c|}
\hline \multirow[b]{2}{*}{$\theta_{\mathrm{AP}}[\operatorname{arcmin}]$} & \multicolumn{3}{|c|}{ LowZ North CGC } & \multicolumn{3}{|c|}{ LowZ South CGC } & \multicolumn{3}{|c|}{ DR13 Group } \\
\hline & $\bar{\tau}\left[10^{-5}\right]$ & $S / N$ & $\chi^{2} /$ d.o.f. & $\bar{\tau}\left[10^{-5}\right]$ & $S / N$ & $\chi^{2} /$ d.o.f. & $\bar{\tau}\left[10^{-5}\right]$ & $S / N$ & $\chi^{2} /$ d.o.f. \\
\hline 3 & $3.7 \pm 2.4$ & $1.56 \sigma$ & 0.52 & $2.0 \pm 4.4$ & $0.47 \sigma$ & 0.31 & $0.9 \pm 1.9$ & $0.44 \sigma$ & 0.77 \\
\hline 4 & $1.9 \pm 2.1$ & $0.91 \sigma$ & 0.66 & $0.5 \pm 3.4$ & $0.14 \sigma$ & 0.55 & $0.6 \pm 1.9$ & $0.30 \sigma$ & 1.00 \\
\hline 5 & $2.9 \pm 2.6$ & $1.10 \sigma$ & 0.80 & $3.6 \pm 4.2$ & $0.86 \sigma$ & 0.55 & $-0.2 \pm 2.2$ & $-0.09 \sigma$ & 0.84 \\
\hline 6 & $3.6 \pm 2.9$ & $1.23 \sigma$ & 0.95 & $3.3 \pm 4.9$ & $0.68 \sigma$ & 0.42 & $2.9 \pm 2.4$ & $1.23 \sigma$ & 0.67 \\
\hline 7 & $5.3 \pm 3.2$ & $1.65 \sigma$ & 0.81 & $3.0 \pm 5.7$ & $0.53 \sigma$ & 0.46 & $4.3 \pm 2.8$ & $1.53 \sigma$ & 0.53 \\
\hline 8 & $4.7 \pm 3.6$ & $1.32 \sigma$ & 0.80 & $3.7 \pm 6.5$ & $0.56 \sigma$ & 0.47 & $3.7 \pm 3.1$ & $1.18 \sigma$ & 0.51 \\
\hline 9 & $3.5 \pm 3.9$ & $0.91 \sigma$ & 0.57 & $0.6 \pm 7.4$ & $0.08 \sigma$ & 0.49 & $1.8 \pm 3.4$ & $0.54 \sigma$ & 0.63 \\
\hline 10 & $2.2 \pm 4.2$ & $0.53 \sigma$ & 0.58 & $0.6 \pm 8.2$ & $0.07 \sigma$ & 0.61 & $1.3 \pm 3.6$ & $0.37 \sigma$ & 0.57 \\
\hline 11 & $2.0 \pm 4.6$ & $0.43 \sigma$ & 0.58 & $-0.8 \pm 8.7$ & $-0.10 \sigma$ & 0.75 & $2.4 \pm 3.7$ & $0.65 \sigma$ & 0.42 \\
\hline
\end{tabular}

$$
U(\theta)=\frac{1}{\pi \theta_{\mathrm{AP}}^{2}} \times \begin{cases}1 & \left(0<\theta<\theta_{\mathrm{AP}}\right) \\ -1 & \left(\theta_{\mathrm{AP}}<\theta<\sqrt{2} \theta_{\mathrm{AP}}\right),\end{cases}
$$

Then, in Fourier space, $U\left(\ell \theta_{\mathrm{AP}}\right)$ is expressed as

$$
U\left(x=\ell \theta_{\mathrm{AP}}\right)=2\left[W_{\text {top }}(x)-W_{\text {top }}(\sqrt{2} x)\right],
$$

where $W_{\text {top }}(x)=2 J_{1}(x) / x$ is the top-hat smoothing window function expressed using $J_{1}(x)$, which is the first order Bessel function of the first kind [42]. In the above $B(\vec{e})=$ $e^{-\sigma_{\mathrm{B}}^{2} \ell^{2} / 2}$ is the Planck beam function in Fourier space, where $\sigma_{\mathrm{B}}=\mathrm{FWHM} / \sqrt{8 \ln 2}$. The FWHM for the Planck 2D-ILC CMB map we used is 5 arcmin.

$N(\vec{\ell})$ describes the gas profile in Fourier space. We assume that the gas profile can be expressed as a projected Gaussian profile. In Fourier space, $N(\vec{\ell})$ is expressed as

$$
N(\ell)=\exp \left[-\frac{\ell^{2} \sigma_{\mathrm{R}}^{2}}{2}\right],
$$

where $\sigma_{\mathrm{R}}$ is the characteristic radius. A typical value of $\sigma_{\mathrm{R}}$ can be estimated from the halo radius $R$ divided by the angular diameter distance $D_{\mathrm{A}}: \sigma_{\mathrm{R}}=R / D_{\mathrm{A}}$. The halos can be identified as the spherical region where the mean density within the radius $R$ is $\Delta \rho_{\text {crit }} E^{2}(z)$, in which $\Delta$ is an experimental constant, $\rho_{\text {crit }}=2.775 h^{2} \times 10^{11} \mathrm{M}_{\odot} \mathrm{Mpc}^{-3}$ is the critical density of the Universe today, and $E(z)=\sqrt{\Omega_{\mathrm{m}}(1+z)^{3}+\Omega_{\Lambda}}$. If $\Delta=200$, the radius $R$ is close to the virial radius, and $\sigma_{\mathrm{R}}$ is close to the virial angular size $\theta_{200}$,

$$
\sigma_{\mathrm{R}}=\theta_{200}=\frac{1}{D_{\mathrm{A}}}\left(\frac{3}{4 \pi} \frac{M_{\mathrm{h}}}{\Delta \rho_{\mathrm{crit}} E^{2}(z)}\right)^{1 / 3} .
$$

We assign $M_{\mathrm{h}}$ and $z_{\text {eff }}$ as free parameters and fit $\bar{\tau}_{\mathrm{m}}\left(\theta_{\mathrm{AP}}\right)$ to the measurements by minimizing the least-squares function,

$$
\chi^{2}=\sum_{i}\left(\bar{\tau}_{\mathrm{m}}\left(\theta_{\mathrm{AP}, i}\right)-\bar{\tau}\left(\theta_{\mathrm{AP}, i}\right)\right)^{2} / \sigma_{\bar{\tau}}^{2}\left(\theta_{\mathrm{AP}, i}\right)
$$

We do not fit to the LowZ South CGC sample due to the lack of significance in measuring $\bar{\tau}$ with this sample. The joint probability of $z_{\text {eff }}$ and $\log M_{\mathrm{h}}$ constrained with the measurements of the LowZ North CGC and DR13 Group are shown in Fig. 8. The filled inner and empty outer contours represent the $68 \%$ and $95 \%$ C.L., respectively. The squares with dashed cross lines indicate the best-fit

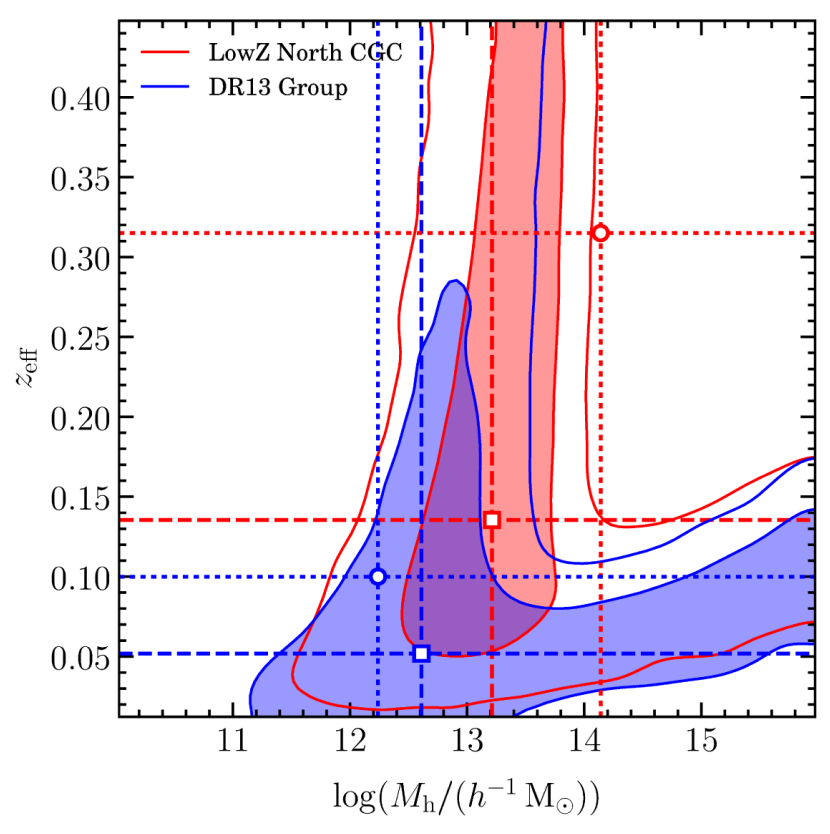

FIG. 8. The joint probability of $z_{\text {eff }}$ and $\log M_{\mathrm{h}}$ constrained with the measured $\bar{\tau}$ of LowZ North CGC (red) and DR13 Group (blue). The filled inner and empty outer contours represent the $68 \%$ and $95 \%$ C.L., respectively. The $68 \%$ C.L. contour for DR13 Group goes beyond the parameter ranges of the plot. The squares with dashed cross lines indicate the best-fit values of $\left[z_{\text {eff }}=0.15, \log M_{\mathrm{h}}=13.24\right]$ for LowZ North CGC and $\left[z_{\text {eff }}=0.05, \log M_{\mathrm{h}}=12.58\right]$ for DR13 Group. The circles with dotted cross lines indicate the median redshift and halo mass of the respective catalogues. 
values. For LowZ North CGC the best-fit values are $\left[z_{\mathrm{eff}}=0.15, \log M_{\mathrm{h}}=13.24\right]$, while for the DR13 Group the best-fit values are $\left[z_{\text {eff }}=0.05, \log M_{\mathrm{h}}=12.58\right]$. The median redshift and halo mass of the catalogue are also shown in Fig. 8 as the circles with dotted cross lines. For Group DR13 the median values are close to the best-fit values and fall within the $68 \%$ C.L. For the LowZ North CGC, the median values fall slightly outside the $2 \sigma$ contour, which implies that the low-redshift and low-mass samples of LowZ North CGC contribute more to the pairwise kSZ signal. We will discuss the mass dependency further in Sec. VE. The theoretical prediction of $\bar{\tau}_{\mathrm{m}}\left(\theta_{\mathrm{AP}}\right)$ using the best-fit $z_{\text {eff }}$ and $\log M_{\mathrm{h}}$ values are shown as the solid lines in Fig. 7.

\section{The mean optical depth $\overline{\boldsymbol{\tau}}$}

We quote the peak value of $\bar{\tau}$ at 7 arcmin as the best-fit values,

$$
\begin{array}{ll}
\bar{\tau}=(0.53 \pm 0.32) \times 10^{-4}(1.65 \sigma) & \text { LowZ North CGC; } \\
\bar{\tau}=(0.30 \pm 0.57) \times 10^{-4}(0.53 \sigma) & \text { LowZ South CGC; } \\
\bar{\tau}=(0.43 \pm 0.28) \times 10^{-4}(1.53 \sigma) & \text { DR13 Group. }
\end{array}
$$

These results are consistent with the measurements in Fourier space with the same catalogue [14], which used the density-weighted pairwise kSZ estimator. By applying the analysis in Fourier space, the method in [14] avoids the correlations between different $k$-bins, thus achieving slightly higher significance.

The mean optical depth is also measured with different CMB maps. De Bernardis et al. [7] reports the results of $\bar{\tau}=(1.46 \pm 0.36) \times 10^{-4}$, using the ACT CMB map and BOSS DR11 galaxy catalogue. The angular resolution of the ACT CMB map is much higher than Planck CMB maps. In their work, the 1.8 arcmin AP filter is applied to the ACT CMB map. Meanwhile, in order to avoid the systematic effect of the less massive clusters, only the 20000 most luminous sources are selected for the analysis. Even more massive clusters are used in the analysis of [10], who report the measurement of $\bar{\tau}=(3.75 \pm 0.89) \times 10^{-3}$ by using the SPT CMB map. Instead of the AP filter, the matched filter is used in the analysis, with filter size of 0.5 arcmin. Because of the small radius of the filter, the mean optical depth reported in [10] is more sensitive to the central region of halos, where the gas density is high compared to the outer regions. Figure 9 shows the histogram of the virial angular size, $\theta_{200}$, of each catalogue used in our study. The virial angular sizes are estimated via Eq. (25). The dotted vertical line shows the filter radius of 7 arcmin. The filter size is large compared to the halo virial radius, which means that the measured mean optical depth is averaged over large regions around the halo center. As a result, our measured mean optical depth is lower. However,

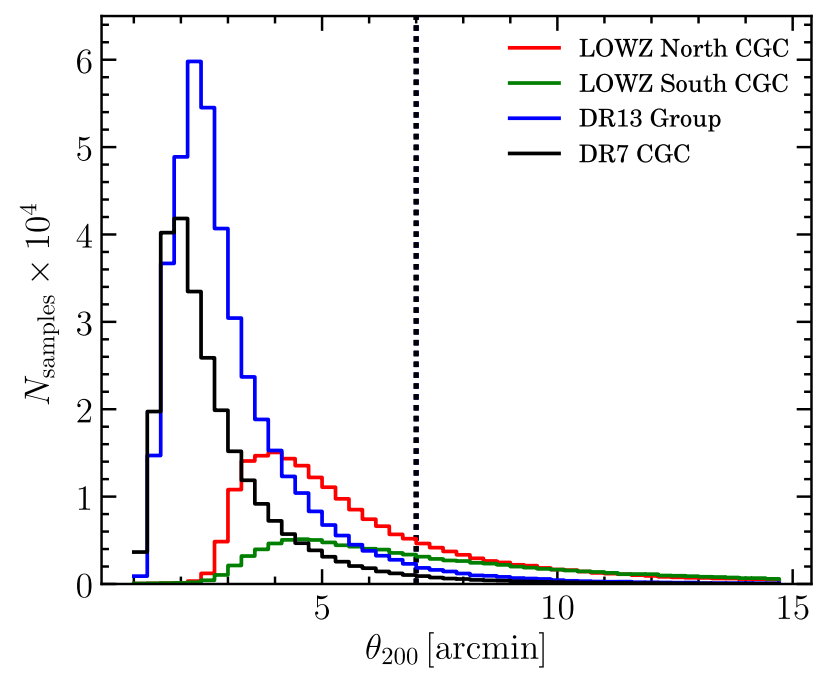

FIG. 9. The $\theta_{200}$ distribution of the galaxy catalogues. The black dotted vertical line indicates $\theta_{200}=7 \mathrm{arcmin}$.

our measurements of $\bar{\tau}$ are consistent with previous analyses that used Planck CMB maps [14,27].

\section{E. Mass dependence}

We split the DR13 Group catalogue into lower and higher halo mass catalogues according to the median value of the halo mass, $\log _{10}\left(M_{\mathrm{h}}^{\text {median }} /\left(h^{-1} \mathrm{M}_{\odot}\right)\right)=12.24$. By using the same pairwise estimator, we measured the mean optical depth with the lower and higher halo mass catalogues.

The top panel of Fig. 10 shows the results with the DR13 Group lower mass (red), higher mass (green) and total (blue) catalogues. Since each subcatalogue contains fewer samples, the errors are larger compared to the full sample. However, for the subcatalogue of lower halo masses, we measure the pairwise kSZ signal at $1 \sigma$ C.L. The best-fit mean optical depth of the lower mass halo catalogue is slightly smaller than that of the higher mass halo catalogue, but still consistent within the $1 \sigma$ error.

Similarly, we also split the LowZ North CGC catalogue into lower and higher halo mass catalogues. The halo mass of the CGC samples are estimated according to the stellar-tohalo mass relation (SHMR) provided by Leauthaud et al. [43], and the stellar mass is obtained by matching the galaxy catalogue with the Portsmouth SED-fit DR12 stellar mass catalogue [44] and the GALEX-SDSS-WISE Legacy Catalog (GSWLC) [45]. The galaxies without matched stellar mass information are ignored in the mass-dependence analysis. The median value of the LowZ North CGC halo mass is $M_{\mathrm{h}}^{\text {median }}=10^{14.14} h^{-1} \mathrm{M}_{\odot}$. More than half of the sources in the LowZ North CGC catalogue are located in halos with masses over $10^{14} h^{-1} \mathbf{M}_{\odot}$. The bottom panel of Fig. 10 shows the results of each subcatalogue for LowZ North CGC. We found that the higher mass subcatalogue lacks a measurement of the pairwise $\mathrm{kSZ}$ signal, due to the high redshift range of the high-mass halos. 

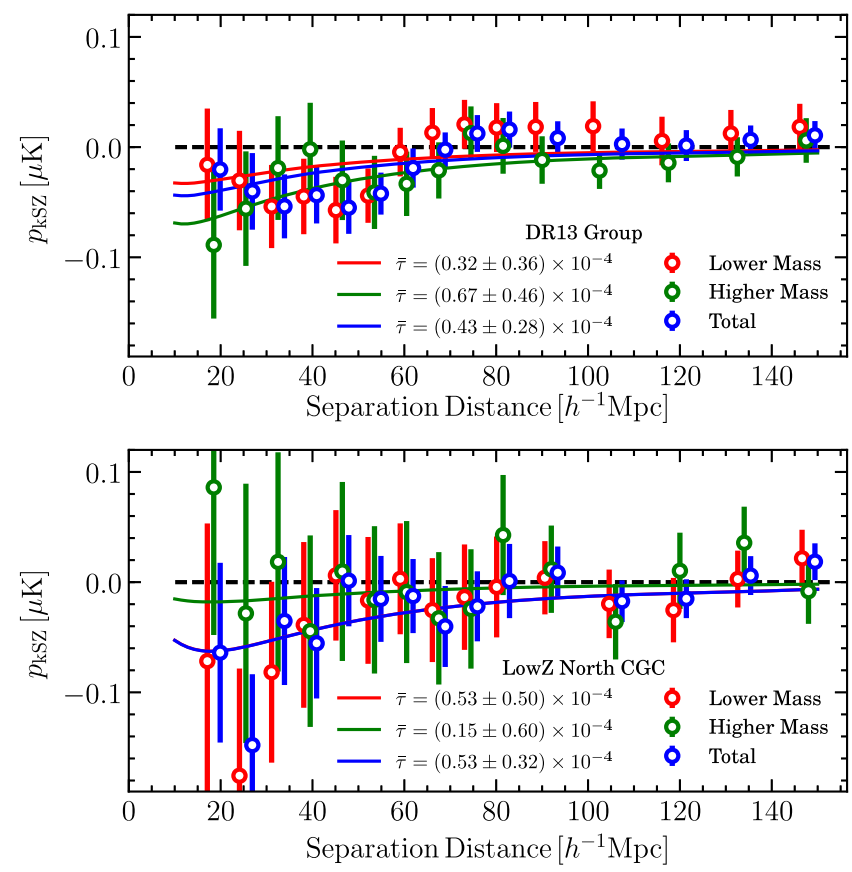

FIG. 10. The measured $\mathrm{kSZ}$ pairwise momentum with different halo mass bins. The catalogues are split into higher and lower halo mass subcatalogues relative to the median value. The upper panel shows the results of the DR13 Group catalogue with median halo mass of $10^{12.24} h^{-1} \mathrm{M}_{\odot}$. The lower panel shows the results of LowZ North CGC with median halo mass of $10^{14.14} h^{-1} \mathrm{M}_{\odot}$.

Compared to the standard LRG criteria used in SDSS-I/II, the LowZ selection includes a bright magnitude cut, which excludes a significant number of low-redshift blue galaxies, but also excludes a fraction of bright galaxies in low-redshift massive clusters [46]. Figure 11 shows the redshift distribution of the lower halo mass, higher halo mass and total LowZ North CGC subcatalogues. It is clear

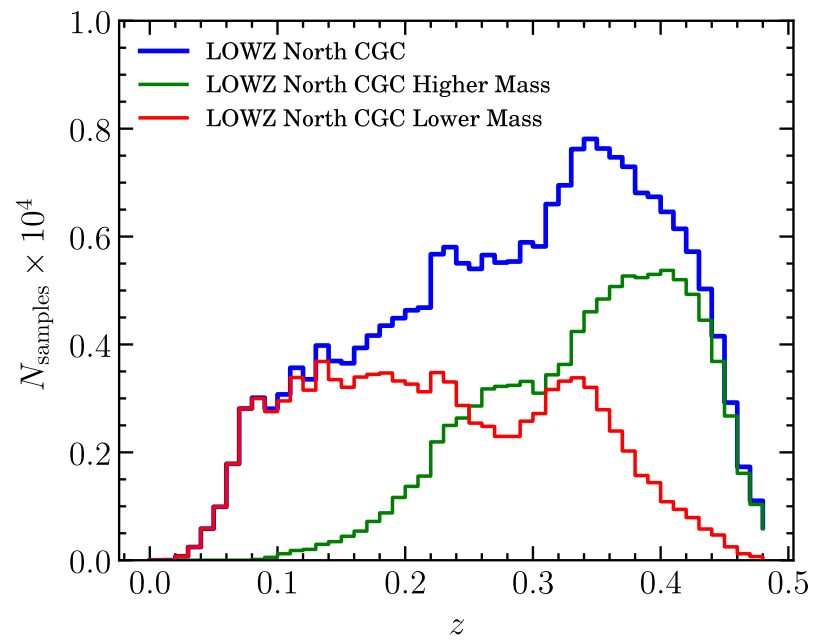

FIG. 11. The redshift distributions of the higher/lower halo mass subcatalogues of LowZ North CGC. that the distribution of high-mass halos is skewed towards higher redshifts.

In order to check whether the pairwise $\mathrm{kSZ}$ signal is dominated by the low-redshift samples, we split the LowZ North CGC catalogue into two redshift subcatalogues relative to the median redshift, $z_{\text {median }}=0.315$. With the same pairwise kSZ estimator we find that, with the lowerredshift subcatalogue, the mean optical depth is $\bar{\tau}=$ $(0.60 \pm 0.43) \times 10^{-4}$, and with the higher-redshift subcatalogue it is $\bar{\tau}=(0.28 \pm 0.58) \times 10^{-4}$. The results for $\bar{\tau}$ using this redshift split follow a similar trend to the measured $\bar{\tau}$ values for the LowZ North CGC lower and higher halo mass splits.

\section{CONCLUSIONS}

We presented the measurements of the pairwise kSZ momentum using the Planck 2D- ILC CMB map, which by construction has completely projected out the tSZ signal, and the Central Galaxy Catalogue (CGC) samples and group catalogue from BOSS DR12 and DR13. The CGC is constructed by selecting the isolated galaxies from the LowZ North/South catalogue of BOSS DR12. The group catalogue we used is based on the halo-based group finder developed by [35] and recently updated with the SDSS DR13 Northern Galactic Cap catalogue.

We used the pair-weighted pairwise kSZ estimator and the AP filter to calculate the signal. The analysis was mainly focused on constraining the mean optical depth $\bar{\tau}$. We first explored the AP filter radius size dependence of the measurements and find that the radius of 7 arcmin gives the maximum detection for $\bar{\tau}$. The results for the three catalogues are

$\begin{array}{ll}\bar{\tau}=(0.53 \pm 0.32) \times 10^{-4}(1.65 \sigma) & \text { LowZ North CGC; } \\ \bar{\tau}=(0.30 \pm 0.57) \times 10^{-4}(0.53 \sigma) & \text { LowZ South CGC; } \\ \bar{\tau}=(0.43 \pm 0.28) \times 10^{-4}(1.53 \sigma) & \text { DR13 Group. }\end{array}$

We showed that the measured values of $\bar{\tau}$ are roughly consistent with the model values of $\bar{\tau}$ obtained using the median values of the halo mass and redshift of each catalogue.

Finally, we investigated the halo mass dependence by splitting the group catalogue and LowZ North CGC into higher and lower halo mass subcatalogues, according to their median halo mass. The group catalogue has most of the sources located in low-mass halos, and the maximum halo mass for the lower-mass group subcatalogue is approximately $10^{12} h^{-1} \mathrm{M}_{\odot}$. We achieved a $1 \sigma$ C.L. detection with such a low-mass catalogue. In comparison, the galaxies in LowZ North CGC are mainly located in highmass halos, with a median value of $10^{14} h^{-1} \mathrm{M}_{\odot}$. We achieved a similar detection with the lower mass LowZ North CGC subcatalogue, compared to the full catalogue, but no detection with the higher mass LowZ North CGC 
subcatalogue. This is because the galaxy samples located in high mass halos are at higher redshifts, which make a smaller contribution to the total pairwise $\mathrm{kSZ}$ signals.

The study we performed here provides a viable method of probing the gas associated with central galaxies, which is an effective way to quantify the baryon fraction of these galaxies. We will perform such studies with future data when SDSS-IV data is released.

\section{ACKNOWLEDGMENTS}

We thank Dr. Carlos Hernández-Monteagudo for crosschecking the results with DR7 CGC samples, Dr. Yu-Ting Wang for the discussion on the BOSS LowZ/CMASS catalogues, Dr. You-Gang Wang for the discussion on the
DR13 Group Catalogue, Dr. Zhi-Gang Li for the discussion on the error estimation, and Dr. Ming Li for the discussion on kSZ measurements and CMB simulations. Y. C. L. and Y.Z.M. acknowledge the support from the National Research Foundation of South Africa with Grant Nos. 105925 and 104800. M. R. acknowledges funding from the European Research Council Starting Consolidator Grant No. 307209. Computations were performed on the Hippo supercomputer at UKZN and the GPC supercomputer at the SciNet HPC Consortium. SciNet is funded by the Canada Foundation for Innovation under the auspices of Compute Canada, the Government of Ontario, Ontario Research Fund-Research Excellence, and the University of Toronto.
[1] R. A. Sunyaev and Y. B. Zeldovich, Comments Astrophys. Space Phys. 4, 173 (1972).

[2] R. A. Sunyaev and I. B. Zeldovich, Mon. Not. R. Astron. Soc. 190, 413 (1980).

[3] N. Hand, G. E. Addison, E. Aubourg, N. Battaglia, E. S. Battistelli, D. Bizyaev, J. R. Bond, H. Brewington, J. Brinkmann, B. R. Brown et al., Phys. Rev. Lett. 109, 041101 (2012).

[4] P. G. Ferreira, R. Juszkiewicz, H. A. Feldman, M. Davis, and A. H. Jaffe, Astrophys. J. Lett. 515, L1 (1999).

[5] D. S. Swetz, P. A. R. Ade, M. Amiri, J. W. Appel, E. S. Battistelli, B. Burger, J. Chervenak, M. J. Devlin, S. R. Dicker, W. B. Doriese et al., Astrophys. J. Suppl. Ser. 194, 41 (2011).

[6] C. P. Ahn, R. Alexandroff, C. Allende Prieto, S. F. Anderson, T. Anderton, B. H. Andrews, É. Aubourg, S. Bailey, E. Balbinot, R. Barnes et al., Astrophys. J. Suppl. Ser. 203, 21 (2012).

[7] F. De Bernardis, S. Aiola, E. M. Vavagiakis, N. Battaglia, M. D. Niemack, J. Beall, D. T. Becker, J. R. Bond, E. Calabrese, H. Cho et al., J. Cosmol. Astropart. Phys. 3 (2017) 008.

[8] P. A. R. Ade, N. Aghanim, M. Arnaud, M. Ashdown, E. Aubourg, J. Aumont, C. Baccigalupi, A. J. Banday, R. B. Barreiro et al. (Planck Collaboration), Astron. Astrophys. 586, A140 (2016).

[9] M. R. Blanton, D. J. Schlegel, M. A. Strauss, J. Brinkmann, D. Finkbeiner, M. Fukugita, J. E. Gunn, D. W. Hogg, Ž. Ivezić, G. R. Knapp et al., Astron. J. 129, 2562 (2005).

[10] B. Soergel, S. Flender, K. T. Story, L. Bleem, T. Giannantonio, G. Efstathiou, E. Rykoff, B. A. Benson, T. Crawford, S. Dodelson et al., Mon. Not. R. Astron. Soc. 461, 3172 (2016).

[11] T. Abbott, F. B. Abdalla, J. Aleksić, S. Allam, A. Amara, D. Bacon, E. Balbinot, M. Banerji, K. Bechtol et al. (Dark Energy Survey Collaboration), Mon. Not. R. Astron. Soc. 460, 1270 (2016).
[12] E. M. George, C. L. Reichardt, K. A. Aird, B. A. Benson, L. E. Bleem, J. E. Carlstrom, C. L. Chang, H.-M. Cho, T. M. Crawford, A. T. Crites et al., Astrophys. J. 799, 177 (2015).

[13] N. S. Sugiyama, T. Okumura, and D. N. Spergel, J. Cosmol. Astropart. Phys. 7 (2016) 001.

[14] N. S. Sugiyama, T. Okumura, and D. N. Spergel, arXiv: 1705.07449 .

[15] J. C. Hill, S. Ferraro, N. Battaglia, J. Liu, and D. N. Spergel, Phys. Rev. Lett. 117, 051301 (2016).

[16] S. Ferraro, J. C. Hill, N. Battaglia, J. Liu, and D. N. Spergel, Phys. Rev. D 94, 123526 (2016).

[17] C. L. Bennett, D. Larson, J. L. Weiland, N. Jarosik, G. Hinshaw, N. Odegard, K. M. Smith, R. S. Hill, B. Gold, M. Halpern et al., Astrophys. J. Suppl. Ser. 208, 20 (2013).

[18] E. L. Wright, P. R. M. Eisenhardt, A. K. Mainzer, M. E. Ressler, R. M. Cutri, T. Jarrett, J. D. Kirkpatrick, D. Padgett, R. S. McMillan, M. Skrutskie et al., Astron. J. 140, 1868 (2010).

[19] N. Aghanim, Y. Akrami, M. Ashdown, J. Aumont, C. Baccigalupi, M. Ballardini, A. J. Banday, R. B. Barreiro, N. Bartolo et al. (Planck Collaboration), arXiv:1707.00132.

[20] S. DeDeo, D. N. Spergel, and H. Trac, arXiv:astro-ph/ 0511060 .

[21] C. Hernández-Monteagudo, L. Verde, R. Jimenez, and D. N. Spergel, Astrophys. J. 643, 598 (2006).

[22] S. Bhattacharya and A. Kosowsky, Astrophys. J. Lett. 659, L83 (2007).

[23] S. Bhattacharya and A. Kosowsky, Phys. Rev. D 77, 083004 (2008).

[24] Y.-Z. Ma and G.-B. Zhao, Phys. Lett. B 735, 402 (2014).

[25] E.-M. Mueller, F. de Bernardis, R. Bean, and M. D. Niemack, Astrophys. J. 808, 47 (2015).

[26] A. Kosowsky and S. Bhattacharya, Phys. Rev. D 80, 062003 (2009).

[27] C. Hernández-Monteagudo, Y.-Z. Ma, F. S. Kitaura, W. Wang, R. Génova-Santos, J. Macías-Pérez, and D. Herranz, Phys. Rev. Lett. 115, 191301 (2015). 
[28] E.-M. Mueller, F. de Bernardis, R. Bean, and M.D. Niemack, Phys. Rev. D 92, 063501 (2015).

[29] M. Remazeilles, J. Delabrouille, and J.-F. Cardoso, Mon. Not. R. Astron. Soc. 410, 2481 (2011).

[30] P. A. R. Ade, N. Aghanim, M. Arnaud, M. Ashdown, J. Aumont, C. Baccigalupi, A. J. Banday, R. B. Barreiro, J. G. Bartlett et al. (Planck Collaboration), Astron. Astrophys. 594, A13 (2016).

[31] J. Delabrouille, J.-F. Cardoso, M. Le Jeune, M. Betoule, G. Fay, and F. Guilloux, Astron. Astrophys. 493, 835 (2009).

[32] S. Basak and J. Delabrouille, Mon. Not. R. Astron. Soc. 419, 1163 (2012).

[33] B. Reid, S. Ho, N. Padmanabhan, W. J. Percival, J. Tinker, R. Tojeiro, M. White, D. J. Eisenstein, C. Maraston, A. J. Ross et al., Mon. Not. R. Astron. Soc. 455, 1553 (2016).

[34] S. H. Lim, H. J. Mo, Y. Lu, H. Wang, and X. Yang, Mon. Not. R. Astron. Soc. 470, 2982 (2017).

[35] X. Yang, H. J. Mo, F. C. van den Bosch, and Y. P. Jing, Mon. Not. R. Astron. Soc. 356, 1293 (2005).

[36] M. Davis and P. J. E. Peebles, Astrophys. J. Suppl. Ser. 34, 425 (1977).

[37] H. J. Mo and S. D. M. White, Mon. Not. R. Astron. Soc. 336, 112 (2002).
[38] J. L. Tinker, B. E. Robertson, A. V. Kravtsov, A. Klypin, M. S. Warren, G. Yepes, and S. Gottlöber, Astrophys. J. 724, 878 (2010).

[39] T. Okumura, U. Seljak, Z. Vlah, and V. Desjacques, J. Cosmol. Astropart. Phys. 5 (2014) 003.

[40] B. Efron, The Jackknife, the Bootstrap and Other Resampling Plans (Society for Industrial and Applied Mathematics, Philadelphia, 1982).

[41] J. Hartlap, P. Simon, and P. Schneider, Astron. Astrophys. 464, 399 (2007).

[42] D. Alonso, T. Louis, P. Bull, and P. G. Ferreira, Phys. Rev. D 94, 043522 (2016).

[43] A. Leauthaud, J. Tinker, K. Bundy, P. S. Behroozi, R. Massey, J. Rhodes, M. R. George, J.-P. Kneib, A. Benson, R. H. Wechsler et al., Astrophys. J. 744, 159 (2012).

[44] C. Maraston, J. Pforr, B. M. Henriques, D. Thomas, D. Wake, J. R. Brownstein, D. Capozzi, J. Tinker, K. Bundy, R. A. Skibba et al., Mon. Not. R. Astron. Soc. 435, 2764 (2013).

[45] S. Salim, J. C. Lee, S. Janowiecki, E. da Cunha, M. Dickinson, M. Boquien, D. Burgarella, J. J. Salzer, and S. Charlot, Astrophys. J. Suppl. Ser. 227, 2 (2016).

[46] H. Hoshino, A. Leauthaud, C. Lackner, C. Hikage, E. Rozo, E. Rykoff, R. Mandelbaum, S. More, A. More, S. Saito et al., Mon. Not. R. Astron. Soc. 452, 998 (2015). 